\title{
Einstein Metrics in Complex Geometry: An Introduction
}

\author{
Toshiki Mabuchi
}

Based on Calabi's pioneering work, systematic studies of EinsteinKähler metrics commenced in mid-seventies with the affirmative solution of Calabi's conjecture by Aubin [2] and Yau [23]. Nowadays, via efforts of Yau himself and also other mathematicians, such metrics are shown to play by far important roles not only in differential geometry but also in algebraic geometry. Moreover, in eighties, the concept of EinsteinHermitian metrics was introduced by S. Kobayashi (cf. [14]), and the resulting analogy of Calabi's conjecture to vector bundle cases, known as Hitchin-Kobayashi's conjecture, was solved affirmatively by Donaldson [9], [10], Uhlenbeck and Yau [22]. This now allows us to make differential-geometric studies of moduli spaces of vector bundles.

In view of these facts, we shall devote Volume 18-II to surveys of recent progress on the study of these metrics. By organizing three working groups, we divided the whole subjects into three categories:

(1) "Einstein-Kähler metrics with positive Ricci curvature" by Futaki, Mabuchi and Sakane.

(2) "Einstein-Kähler metrics with non-positive Ricci curvature" by Bando, Enoki, R. Kobayashi and Sugiyama.

(3) "Yang-Mills connections and Einstein-Hermitian metrics" by Itoh and Nakajima.

All of these are intended to be highly of expository nature but I believe that some particular places therein are written as original works. Now, to provide an introduction to subsequent surveys, we shall briefly discuss basic facts on Einstein metrics in complex geometry.

\section{§1. Einstein-Kähler metrics and Calabi's Conjecture}

Let $X$ be an $n$-dimensional compact connected complex manifold endowed with a Kähler form $\omega$. Then the corresponding Ricci form

$$
\operatorname{Ric}(\omega)=\sqrt{-1} \partial \bar{\partial} \log \omega^{n}
$$


represents the de Rham cohomology class $2 \pi c_{1}(X)_{\mathbb{R}}$. Recall that $\omega$ is called an Einstein-Kähler form if $\operatorname{Ric}(\omega)=\lambda \omega$ for some real constant $\lambda$. Moreover, $\operatorname{Ric}(\omega)$ does not vary even if we replace $\omega$ by its positive constant multiple. Therefore, for an Einstein-Kähler form $\omega$, such a replacement enables us to assume that $\lambda$ above is either -1 or 0 or 1 . Hence the following three cases are possible.

(1) $\lambda=-1:-\omega$ represents $2 \pi c_{1}(X)_{\mathbb{R}}$, so that $c_{1}(X)_{\mathbb{R}}<0$.

(2) $\lambda=0: \quad \omega$ is Ricci-flat, so that $c_{1}(X)_{\mathbb{R}}=0$.

(3) $\lambda=1: \quad \omega$ represents $2 \pi c_{1}(X)_{\mathbb{R}}$, so that $c_{1}(X)_{\mathbb{R}}>0$.

There arises naturally the question whether or not the converse is true. Recall the following well-known conjecture posed by Calabi:

\section{Calabi's Conjecture.}

(a) If $c_{1}(X)_{\mathbb{R}}<0$, then $X$ admits a unique Einstein-Kähler form $\omega$ satisfying $\operatorname{Ric}(\omega)=-\omega$.

(b) If $c_{1}(X)_{\mathbb{R}}=0$, then each Kähler class on $X$ contains a unique Ricci-flat Kähler form.

(c) If $c_{1}(X)_{\mathbb{R}}>0$ and $h^{0}(X, \mathcal{O}(T M))=0$, then $X$ admits a unique Einstein-Kähler form $\omega$ satisfying $\operatorname{Ric}(\omega)=\omega$.

Most of this conjecture has already been proved affirmatively. For instance, the uniqueness is always true; see Calabi [7] for (a) and (b), and see Bando and Mabuchi [4] for (c). The existence, except (c), is established by Aubin [2] and Yau [23]: Namely, Aubin solved (a) affirmatively by treating a Monge-Ampère equation, while a little afterwards, Yau gave a complete affirmative answer to (b), as well as (a), by making systematic studies of such equations. However, (c) is still open unless either $\operatorname{dim}_{\mathbb{C}} X \leq 2$ or $X$ admits a suitable finite symmetry (see, Siu [18], Tian [20], Tian and Yau [21]).

\section{§. Einstein-Kähler metrics for compact Riemann surfaces}

Let us now give examples of Einstein-Kähler metrics. For simplicity, assume $\operatorname{dim}_{\mathbb{C}} X=1$, so that $X$ is a compact Riemann surface of some genus $p$. Put $\Gamma:=\pi_{1}(X)$. Moreover, let $\gamma$ denote the positive generator of the cohomology group $H^{2}(X, \mathbb{Z}) \cong \mathbb{Z}$. Then $X$ carries an EinsteinKähler metric $g_{X}$, unique up to the action of $\operatorname{Aut}^{0}(X)$, such that the associated Einstein-Kähler form (denoted also by $g_{X}$ ) represents the class $(2 p-2) \gamma, \gamma$, or $2 \gamma$, according as $c_{1}(X)_{\mathbb{R}}$ is negative, zero, or positive:

(1) $c_{1}(X)_{\mathbb{B}}<0$, i.e., $X=\Delta / \Gamma$, where $\Gamma$ acts biholomorphically and freely on $\Delta=\{z \in \mathbb{C} ;|z|<1\}$. Then the pull-back of $g_{X}$ to $\Delta$ 
is nothing but the Poincaré metric

$$
\frac{2}{\left(1-|z|^{2}\right)^{2}} \sqrt{-1} d z \wedge d \bar{z}=\sqrt{-1} \bar{\partial} \partial\left\{\log \frac{\left(1-|z|^{2}\right)^{2}}{2}\right\} \text {. }
$$

(2) $c_{1}(X)_{\mathbb{R}}=0$, i.e., $X=\mathbb{C} / \Gamma$, where $\Gamma$ is a lattice $\mathbb{Z}+\mathbb{Z} \tau$ for some $\tau \in \mathbb{C}$ with $\operatorname{Im} \tau>0$. Then the pull-back of $g_{X}$ to $\mathbb{C}$ is the translation-invariant flat metric

$$
(2 \operatorname{Im} \tau)^{-1} \sqrt{-1} d z \wedge d \bar{z}
$$

(3) $c_{1}(X)_{\mathbb{R}}>0$, i.e., $X=\mathbb{P}^{1}(\mathbb{C})=\left\{\left(z_{1}: z_{2}\right)\right\}$. By setting $z:=$ $z_{1} / z_{2}$, we can express $g_{X}$ as the Fubini-Study metric

$$
\frac{2}{\left(1+|z|^{2}\right)^{2}} \sqrt{-1} d z \wedge d \bar{z}=\sqrt{-1} \partial \bar{\partial} \log \left\{\frac{\left(1+|z|^{2}\right)^{2}}{2}\right\} \text {. }
$$

Thus, we see that $\operatorname{Ric}\left(g_{X}\right)$ is nothing but $-g_{X}$, 0 , or $g_{X}$, according as $c_{1}(X)_{\mathbb{R}}$ is negative, zero, or positive.

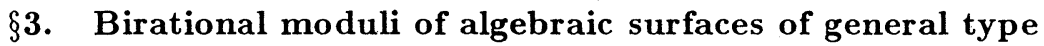

Let $\mathcal{M}$ be the set of all connected nonsingular projective algebraic surfaces (defined over $\mathbb{C}$ ) of general type. For $X, Y \in \mathcal{M}$, we write $X \sim Y$ if $X$ is birational to $Y$. Then the set of equivalence classes

$$
\mathcal{M}_{\text {bir }}:=\mathcal{M} / \sim
$$

is nothing but the birational moduli space of algebraic surfaces of general type. For each $X$ in $\mathcal{M}$, let $[X]$ denote the associated equivalence class in $\mathcal{M}_{\text {bir }}$. Note that $X$ is called a canonically polarized RDP surface if $X$ has ample canonical divisor $K_{X}$ and is in addition free from singularities other than rational double points. Now, let $\mathcal{M}_{\mathrm{RDP}}$ be the set of all canonically polarized RDP surfaces (modulo isomorphisms). We then have a natural bijection

$$
\mathcal{M}_{\mathrm{bir}} \simeq \mathcal{M}_{\mathrm{RDP}}, \quad[X] \leftrightarrow X_{\mathrm{can}}
$$

where $X_{\text {can }}$ denotes the canonical model of $X \in \mathcal{M}$ defined by

$$
X_{\text {can }}=\operatorname{Proj}\left(\oplus_{\nu=0}^{\infty} H^{0}\left(X, \mathcal{O}\left(\nu K_{X}\right)\right)\right)
$$

Let $\left\{\sigma_{0}, \sigma_{1}, \ldots, \sigma_{N}\right\}$ be a $\mathbb{C}$-basis for $H^{0}\left(X, \mathcal{O}\left(5 K_{X}\right)\right)$. Then, more geometrically, $X_{\text {can }}$ is nothing but the image of (cf. Bombieri [6])

$$
\Phi_{5}: X \rightarrow \mathbb{P}^{N}(\mathbb{C}), \quad x \mapsto \Phi_{5}(x):=\left(\sigma_{0}(x): \sigma_{1}(x): \cdots: \sigma_{N}(x)\right) .
$$


Now by R. Kobayashi [12], the results of Aubin [2] and Yau [23] on Calabi's Conjecture are valid also for orbifolds. Recall that rational double singularities are isolated quotient singularities. Hence, every $X \in \mathcal{M}_{\mathrm{RDP}}$ carries an Einstein-Kähler orbifold metric $g_{X}$ uniquely characterized by the identity $\operatorname{Ric}\left(g_{X}\right)=-g_{X}$. Let $\mathcal{M}_{\mathrm{EK}}$ be the set of all EinsteinKähler orbifolds $(X, g)$, modulo holomorphic isometries, such that $X$ is in $\mathcal{M}_{\mathrm{RDP}}$ and that $\operatorname{Ric}(g)=-g$. Then the mapping

$$
\operatorname{pr}_{1}: \mathcal{M}_{\mathrm{EK}} \rightarrow \mathcal{M}_{\mathrm{bir}}, \quad\left(X, g_{X}\right) \mapsto X,
$$

is bijective. The injectiveness of $\mathrm{pr}_{1}$ is straightforward from the uniqueness of Einstein-Kähler metrics, while the surjectiveness of $\mathrm{pr}_{1}$ follows from the existence of Einstein-Kähler metrics for all $X$ in $\mathcal{M}_{\mathrm{RDP}}$. We thus have an identification

$$
\mathcal{M}_{\mathrm{bir}} \simeq \mathcal{M}_{\mathrm{EK}}
$$

between algebraic-geometric $\mathcal{M}_{\text {bir }}$ and differential-geometric $\mathcal{M}_{\mathrm{EK}}$. This identification now allows us to look at $\mathcal{M}_{\text {bir }}$ from differential-geometric viewpoints.

\section{$\S 4$. Uniformization by Miyaoka-Van de Ven-Yau's inequality}

We shall next study another aspect of Einstein-Kähler metrics. For simplicity, let $X$ be a nonsingular irreducible projective algebraic surface defined over $\mathbb{C}$. Recall that Yau's affirmative solution of Calabi's Conjecture gave us some information on the extremal case of Miyaoka-Van de Ven-Yau's inequality:

$$
c_{1}(X)^{2} \leq 3 c_{2}(X) \quad \text { if } c_{1}(X)_{\mathbb{R}}<0 .
$$

Namely, the equality holds if and only if the complex surface obtained as the universal cover of $X$ is just the open unit ball $B^{2}=\left\{\left|z_{1}\right|^{2}+\right.$ $\left.\left|z_{2}\right|^{2}<1\right\}$ in $\mathbb{C}^{2}$. This uniformization result, for instance, enabled Yau to show the uniqueness of complex structure on $\mathbb{P}^{2}(\mathbb{C})$, which solved Severi's conjecture affirmatively. Another important consequence can be obtained by generalizing the uniformization result to ramified cases (see for instance R. Kobayashi [13]). Let $Y$ be a compact complex connected normal surface with a finite number of irreducible reduced curves $D_{i}$, $i \in I$, sitting in $Y$. Associating, to each $i$, either an integer $b_{i} \geq 2$ or $b_{i}=\infty$, we have an effective Weil Q-divisor

$$
D=\Sigma_{i}\left(1-b_{i}^{-1}\right) D_{i} \in \operatorname{Div}_{\mathbb{Q}}(Y) .
$$


Let $Y_{\text {sing }}, D_{\text {sing }}$ be the (possibly empty) set of the singular points in $Y$, $\operatorname{Supp}(D)$, respectively. In particular, $D_{i} \cup D_{j} \subset D_{\text {sing }}$ for all $i, j \in I$ with $i \neq j$. Assume that all points in $Y_{\text {sing }} \cup D_{\text {sing }}$ are log-canonical singularities of $(Y, D)$, i.e., we have a birational morphism $\mu: \tilde{Y} \rightarrow Y$ of a nonsingular projective algebraic surface $\tilde{Y}$ onto $Y$ such that

(1) the set $\mu^{-1}\left(Y_{\text {sing }} \cup \operatorname{Supp}(D)\right)$ has only simple normal crossings in the sense that its irreducible components are all nonsingular intersecting transversally.

(2) Let $\tilde{D}$ be the Weil $Q$-divisor obtained from $D$ by replacing each $D_{i}$ with its proper transform $\tilde{D}_{i}$ in $\tilde{Y}$. Write the exceptional set $\mu^{-1}\left(Y_{\text {sing }} \cup D_{\text {sing }}\right)$ as a union $\cup_{\alpha=1}^{k} E_{\alpha}$ of irreducible components. The rational numbers $a_{\alpha}$ defined by the equations

$$
\left(K_{\tilde{Y}}+\tilde{D}+\Sigma_{\alpha} a_{\alpha} E_{\alpha}, E_{\beta}\right)=0, \quad 1 \leq \beta \leq k .
$$

satisfy $a_{\alpha} \leq 1$ for all $\alpha$.

Then by setting $E:=\Sigma_{\alpha} a_{\alpha} E_{\alpha}$, we have $\mu^{*}\left(K_{Y}+D\right)=K_{\tilde{Y}}+\tilde{D}+E$, and $K_{Y}+D$ is $\mathbb{Q}$-Cartier. Let $m_{0}$ be the smallest positive integer such that $m_{0}\left(K_{Y}+D\right)$ is a Cartier divisor on $Y$. Assume now that $\bar{\kappa}(Y, D)=2$, i.e., the $\log$-canonical model $Y_{\text {can }}:=\operatorname{Proj} \bar{R}(Y, D)$ is a projective algebraic surface, where $\bar{R}(Y, D)$ denotes the graded ring

$$
\bar{R}(Y, D)=\bigoplus_{m=0}^{\infty} H^{0}\left(Y, \mathcal{O}\left(m m_{0}\left(K_{Y}+D\right)\right)\right)
$$

Note here that $Y_{\text {can }}$ is obtained from $Y$ by contracting successively logexceptional curves of the first and second kinds. Let $\psi: Y \rightarrow Y_{\text {can }}$ be the contraction. Take the Zariski decomposition $K_{Y}+D=\left(K_{Y}+D\right)^{+}+$ $\left(K_{Y}+D\right)^{-}$. Then $\left(K_{Y}+D\right)^{+}$is nothing but the divisor $\psi^{*} \psi_{*}\left(K_{Y}+D\right)$. Let $D_{\infty}$ be the union of all $D_{i}(i \in I)$ such that $b_{i}=\infty$. Moreover, let $S$ be the set of singular points of $Y_{\text {can }}$ other than quotient sigularities. Then every point in $S$ is a finite cyclic quotient of either a simple elliptic singularity or a cusp singularity. We now put

$$
\begin{aligned}
Y^{0} & :=Y_{\text {can }}-\left(\psi\left(D_{\infty}\right) \cup S\right), \\
D^{0} & :=\psi(\operatorname{Supp}(D)) \cap Y^{0}
\end{aligned}
$$

We further put $D_{i}^{0}:=\psi\left(D_{i}\right) \cap Y^{0}$ for each $i$, and let $d_{i}$ be the settheoretical number of the points in $D_{i}^{0} \cap\left(Y_{\text {sing }}^{0} \cup D_{\text {sing }}^{0}\right)$. For each point $q$ in $Y_{\text {sing }}^{0} \cup D_{\text {sing }}^{0}$, let $G_{q}$ be the local fundamental group at $q$ of the 
orbifold pair $\left(Y^{0}, D^{0}\right)$. Let $r_{q}$ be the order $\left|G_{q}\right|$ of the group $G_{q}$. Then by setting $P:=\psi_{*}\left(K_{Y}+D\right)$, we have the inequality (cf. [13])

$$
\left(P^{2}\right) \leq 3\left\{e\left(Y^{0}\right)-\Sigma_{i}\left(1-b_{i}^{-1}\right)\left(e\left(D_{i}^{0}\right)-d_{i}\right)-\Sigma_{q}\left(1-r_{q}^{-1}\right)\right\},
$$

where $e(\cdot)$ denotes the Euler number. The equality (called the extremal case of the inequality) holds if and only if there exists a discrete subgroup $\Gamma$ of the group $\operatorname{Aut}\left(B^{2}\right)$ of holomorphic automorphisms of $B^{2}$ such that

(a) $Y^{0}$ is biholomorphic to the ball quotient $B^{2} / \Gamma$;

(b) outside the set $\left(B^{2} / \Gamma\right)_{\text {sing }} \cup D_{\text {sing }}^{0}$, the covering $\rho: B^{2} \rightarrow B^{2} / \Gamma$ is branched exactly over $\left\{D_{i}^{0} ; b_{i} \neq \infty\right\}$ with branch indices $\left\{b_{i}\right\}$.

Interesting examples of ball quotients $Y^{0}=B^{2} / \Gamma$ such that

(1) $Y^{0}=Y_{\text {can }}=\mathbb{P}^{2}(\mathbb{C})$;

(2) $\quad \ell_{i}:=\psi\left(D_{i}\right)$ is a line in $\mathbb{P}^{2}(\mathbb{C})$ for all $i$ with $b_{i} \neq \infty$

were studied by many mathematicians (see for instance Hirzebruch [11]). An essential point in constructing such examples is to find out configurations of lines $\ell_{i} \subset \mathbb{P}^{2}(\mathbb{C})$ with suitable branch indices $b_{i}$ such that the extremal case of the above inequality holds. Once such an example is constructed, the following question arises: Can one find a Fuchsian differential equation on $\mathbb{P}^{2}(\mathbb{C})$ with regular singular points exactly along the lines $\ell_{i}$ ? Suppose yes. Let $\omega_{0}, \omega_{1}, \ldots, \omega_{N}$ be the set of all linearly independent solutions for the equation. Consider the multi-valued map

$$
\tau: \mathbb{P}^{2}(\mathbb{C}) \rightarrow \mathbb{P}^{N}(\mathbb{C}), \quad x \mapsto \tau(x):=\left(\omega_{0}(x): \omega_{1}(x): \cdots: \omega_{N}(x)\right) .
$$

Let $\Gamma$ be the image of the projective monodromy representation

$$
\pi_{1}\left(\mathbb{P}^{2}(\mathbb{C})-\cup_{i} \ell_{i}\right) \rightarrow \operatorname{PGL}(N+1, \mathbb{C}) .
$$

Then we have a generically well-defined map

$$
\tilde{\tau}: \mathbb{P}^{2}(\mathbb{C}) \rightarrow \operatorname{Image}(\tau) / \Gamma
$$

induced by $\tau$. If $\tilde{\tau}$ is nothing but a bijection satisfying Image $(\tau) \cong B^{2}$, then the mapping $\tau$ is regarded as the inverse of $\rho: B^{2} \rightarrow \mathbb{P}^{2}(\mathbb{C})=B^{2} / \Gamma$. This construction is actually possible for several series of examples (see for instance Yoshida [24]). We finally note that examples of Terada [19], Deligne and Mostow [8] are easily understood from a differentialgeometric point of view by checking that they satisfy just the extremal cases of the above inequality. 


\section{$\S 5$. Einstein-Hermitian metrics and stable vector bundles}

Let $E$ be a holomorphic vector bundle, of rank $r$, over a compact connected $n$-dimensional Kähler manifold $(X, \omega)$. For a Hermitian metric $h$ on $E$, the corresponding curvature form is given by

$$
\mathrm{R}(h)=\sqrt{-1} \bar{\partial}\left(h^{-1} \partial h\right) .
$$

We then put

$$
\Lambda \mathrm{R}(h):=\frac{n \mathrm{R}(h) \wedge \omega^{n-1}}{\omega^{n}} \in C^{\infty}(\text { End } E) .
$$

Recall that $h$ is called an Einstein-Hermitian metric if $\Lambda \mathrm{R}(h)=c_{0} \mathrm{id}_{E}$, where $c_{0}=\left(r \int_{X} \omega^{n}\right)^{-1}\left(n \int_{X} c_{1}(E) \wedge \omega^{n-1}\right)$. Moreover, $E$ is said to be stable if the inequality

$$
\frac{\int_{X} c_{1}(\mathcal{S}) \wedge \omega^{n-1}}{\operatorname{rank} \mathcal{S}}<\frac{\int_{X} c_{1}(\mathcal{E}) \wedge \omega^{n-1}}{r}
$$

holds for every coherent subsheaf $\mathcal{S}$ of $\mathcal{O}(E)$ with $0<\operatorname{rank} \mathcal{S}<r$. Let us now assume that $E$ is indecomposable, i.e., $E$ cannot be written as a direct sum of two holomorphic vector bundles of lower ranks. A theorem of S. Kobayashi [14] and Lübke [17] shows that, if $E$ admits an Einstein-Hermitian metric, then $E$ is stable. Hitchin and S. Kobayashi conjectured its converse.

Hitchin-Kobayashi's Conjecture. If $E$ is stable, then $E$ admits an Einstein-Hermitian metric (unique up to constant multiple).

This can be regarded as a vector-bundle version of Calabi's conjecture, and was solved affirmatively by Donaldson [9], [10], Uhlenbeck and Yau [22]. This affirmative solution not only provided us with new methods in the study of moduli spaces of stable vector bundles, but also yielded numerous remarkable results on the differential structures of algebraic surfaces.

\section{$\S 6$. Hyperkähler manifolds}

A complete Riemannian manifold $(X, g)$ is said to be hyperkähler if there exists global differentiable sections $I, J, K$ of $\operatorname{End}(T X)$ such that

(a) $I^{2}=J^{2}=K^{2}=-\mathrm{id}_{T X}$,

(b) $I J=-J I=K, J K=-K J=I, K I=-I K=J$,

(c) $\nabla I=\nabla J=\nabla K=0$, 
where $\nabla$ denotes the covariant derivative naturally induced by the Riemannian metric $g$. Note that every hyperkähler manifold is Ricci-flat (cf. Berger [5]). For instance, every K3-surface $S$ admits a hyperkähler structure $(I, J, K)$ such that

(a) $I$ is the complex structure of $S$,

(b) $\sqrt{-1} \omega_{\mathrm{J}}-\omega_{\mathrm{K}} \in H^{0}\left(S, \mathcal{O}\left(K_{S}\right)\right)$, and $\omega_{\mathrm{I}}$ is a Ricci-flat EinsteinKähler form on $S$, where $\omega_{\mathrm{I}}, \omega_{\mathrm{J}}, \omega_{\mathrm{K}}$ are the real symplectic 2 -forms on $S$ defined respectively by the identities

$$
\omega_{\mathrm{I}}(u, v)=g(I u, v), \omega_{\mathrm{J}}(u, v)=g(J u, v), \omega_{\mathrm{K}}(u, v)=g(K u, v),
$$

with tangent vectors $u, v \in\left(T_{s} S\right)_{\mathbb{C}}, s \in S$.

An open version of a K3-surface is an asymptotically locally Euclidean hyperkähler 4-manifold. Kronheimer [15], [16] recently proved the following:

Theorem. Every asymptotically locally Euclidean hyperkähler 4manifold is diffeomorphic to the minimal resolution of the Kleinian singularity $\mathbb{C}^{2} / \Gamma$ for some finite subgroup $\Gamma$ of $\mathrm{SU}(2)$.

His new viewpoint relates Kleinian singularities to hyperkähler geometry via Mckay's observation. Moreover, recent works of Anderson [1], Bando, Kasue and Nakajima [3] show that, in Gromov's compactification of the moduli space of Ricci-positive Einstein-Kähler surfaces, every boundary point corresponds to a Ricci-positive Einstein-Kähler orbifold. Namely, in the degeneration procedure, a finite number of finite cyclic quotients of asymptotically locally Euclidean hyperkähler 4-orbifolds always bubble off producing the singular points of the limit orbifold. It is expected that some good theory exists also for more complicated singularities such as simple elliptic singularities or simple K3-singularities.

\section{References}

[1] M. Anderson, Ricci curvature bounds and Einstein metrics on compact manifolds, J. Amer. Math. Soc., 2 (1989), 455-490.

[2] T. Aubin, "Nonlinear analysis on manifolds", Springer, Berlin, New York, 1982.

[3] S. Bando, A. Kasue and H. Nakajima, On a construction of coordinates at infinity on manifolds with fast curvature decay and maximal volume growth, Invent. Math., 97 (1989), 313-349.

[4] S. Bando and T. Mabuchi, Uniqueness of Einstein Kähler metrics modulo connected group actions, in "Algebraic Geometry, Sendai, 1985", Adv. Stud. in Pure Math. 10, Kinokuniya, Tokyo and North-Holland, Amsterdam, 1987, pp. 11-40. 
[ 5 ] M. Berger, Sur les groupes d'holonomie des variétés à connexion affine et des variétés riemanniennes, Bull. Soc. Math. France, 83 (1955), 279-330.

[6] E. Bombieri, Canonical models of surfaces of general type, publ. IHES, 42 (1973), 447-495.

[ 7 ] E. Calabi, On Kähler manifolds with vanishing canonical class, in "Algebraic geometry and topology, a symposium in honor of S. Lefschetz", ed. R. H. Fox, D. C. Spencer, A. W. Tucker, Princeton Univ. Press, Princeton, 1957, pp. 78-89.

[8] P. Deligne and G. D. Mostow, Monodromy of hypergeometric functions and non-lattice integral monodromy, Publ. IHES, 63 (1986), 5-106.

[9] S. K. Donaldson, Anti-self-dual Yang-Mills connections over complex algebraic surfaces and stable vector bundles, Proc. London Math. Soc., 50 (1985), 1-26.

[10] - Infinite determinants, stable bundles and curvature, Duke Math. J., 54 (1987), 231-247.

[11] F. Hirzebruch, Arrangements of lines and algebraic surfaces, in "Arithmetic and geometry", ded. I. R. Shafarevich, Progress in Math. 36, Birkhäuser, Boston, Basel, Stuttgart, 1983, pp. 113-140.

[12] R. Kobayashi, Einstein-Kähler $V$-metrics on open Satake $V$-surfaces with isolated quotient singularities, Math. Ann., 272 (1985), 385-398.

[13] Uniformization of complex surfaces, in this volume.

[14] S. Kobayashi, "Differential geometry of complex vector bundles", Publ. Math. Soc. Japan 14, Iwanami and Princeton Univ. Press, Tokyo and Princeton.

[15] P. B. Kronheimer, The construction of ALE spaces as hyper-kähler quotients, J. Differential Geometry, 29 (1989), 665-683.

[16] A Torelli-type theorem for gravitational instantons, J. Differential Geometry, 29 (1989), 685-697.

[17] M. Lübke, Stability of Einstein-Hermitian vector bundles, Manuscripta Math., 42 (1983), 245-257.

[18] Y.-T. Siu, "Lectures on Hermitian-Einstein metrics for stable bundles and Kähler-Einstein metrics", DMV seminar '86, vol. 8, Birkhäuser, Basel, Boston, Stuttgart, 1987.

[19] T. Terada, Quelques propriétes géometrique de domaine de $F_{1}$ et le groupe de tresses colorées, Publ. RIMS, 17 (1981), 95-111.

[20] G. Tian, On Kähler-Einstein metrics on certain Kähler manifolds with $C_{1}(M)>0$, Invent. Math., 89 (1987), 225-246.

[21] G. Tian and S. T. Yau, Kähler-Einstein metrics on complex surfaces with $C_{1}>0$, Commun. Math. Phys., 112 (1987), 175-203.

[22] K. Uhlenbeck and S. T. Yau, On the existence of Hermitian-Yang-Mills connections in stable vector bundles, Commun. Pure and Appl. Math., 39 (1986), 258-293.

[23] S. T. Yau, On the Ricci curvature of a compact Kähler manifold and the complex Monge-Ampère equation I, Commun. Pure and Appl. Math., 
31 (1978), 339-411.

[24] M. Yoshida, "Fuchsian differential equations", Aspects of Mathematics E11, Vieweg, Braunschweig, 1987.

Department of Mathematics, College of General Education, Osaka University,

Toyonaka, Osaka, 560

Japan 